\title{
Design and Reliability Analysis of New Fault-Tolerant Irregular Multistage Interconnection Network
}

\author{
Mamta Ghai \\ Department of Computer \\ Science, \\ Punjabi University, Patiala.
}

\author{
Karamjit Kaur Cheema \\ Department of Computer \\ Science, \\ Thapar University, Patiala.
}

\author{
Vinay Chopra \\ Department of computer \\ science, \\ D.A.V College, Jalandhar.
}

\begin{abstract}
Parallel processing is an efficient form of information processing system, which emphasizes the exploitation of concurrent events in the computing process. To achieve parallel processing it's required to develop more capable and cost-effective systems.In order to operate more efficiently a network is required to handle large amount of traffic. Multi-stage Interconnection Network plays a vital role on the performance of these multiprocessor systems.In this paper an attempt has been made to analyze the characteristics of a new class of irregular fault-tolerant multistage interconnection network named as irregular modified augmented baseline network (IMABN). IMABN can provide 'Full access' capability in presence of multiple faults. The reliability of interconnection networks and their ability to continue operating despite failures are major concerns in determining the overall system performance. In this paper reliability of the proposed IMABN have been calculated and compared in terms of the Upper and Lower bounds of mean time to failure (MTTF). Reliability and Cost study shows that IMABNs achieve a significant improvement over Modified Augmented Baseline Network (MABN).
\end{abstract}

Keywords: Multistage interconnection networks, Cost, Reliability, Irregular modified baseline network.

\section{INTRODUCTION}

With the present state of technology building multiprocessor system with hundreds of processors is feasible vital component of these systems is the interconnection network(IN) that enables the processors to communicate among themselves or with the memory units.Multipath nature of multistage interconnection networks become more popular. Many ways of providing fault-tolerance to multistage interconnection networks(MINs) have been proposed.The basic idea for fault-tolerance is to provide multiple paths between source-destination pair so that alternate paths can been used in case of faults. Sufficient work has been done on the regular type of MINs, but little attention has been paid to the irregular type of MIN.In this paper, a new class of irregular fault-tolerant multistage interconnection network named as irregular modified augmented baseline network(IMABN) is proposed. In this paper we present methods of increasing fault-tolerance of an network by introducing the extra stage. Hence with the additional stage more paths available between each source and destination, as compared to existing network MABN. The proposed Irregular Modified Augmented Baseline
Network (IMABN) is an Modified augmented baseline network (MABN) [10] with additional stage. In an IMABN, there are Six possible paths between any source-destination pair, whereas MABN has only Four. As we will see, IMABNs can achieve general goals for the design of fault-tolerant networks i.e. good reliability, Cost effective, simple control.In the following section structure and design of existing network MABN and proposed network IMABN is described. The Reliability analysis of IMABN is given in section 3. Cost analysis of IMABN is given in a section 4. Finally, some concluding remarks are given in section 5 .

\section{STRUCTURE OF NETWORKS}

\subsection{Construction of MABNs}

To construct an MABN of size N, two identical groups of N/2 sources and N/2 destinations need to be formed first. Each source is linked to both the groups via multiplexers. There is one $4 \times 1$ MUX for each input link of a switch in stage 1 and one 1 x 4 DEMUX for each output link of a switch in stage n-2. MABN consists of two identical sub-networks which are denoted by Gi. Switches A, B, C, D belonging to stage 1 of a sub-network (Gi) form a conjugate subset,switches $\mathrm{A}$ and $\mathrm{B}$ form a conjugate pair, and switches $\mathrm{A}$ and $\mathrm{C}$ form a conjugate loop. An MABN of size $16 \mathrm{X} 16$ is shown in Figure 1.

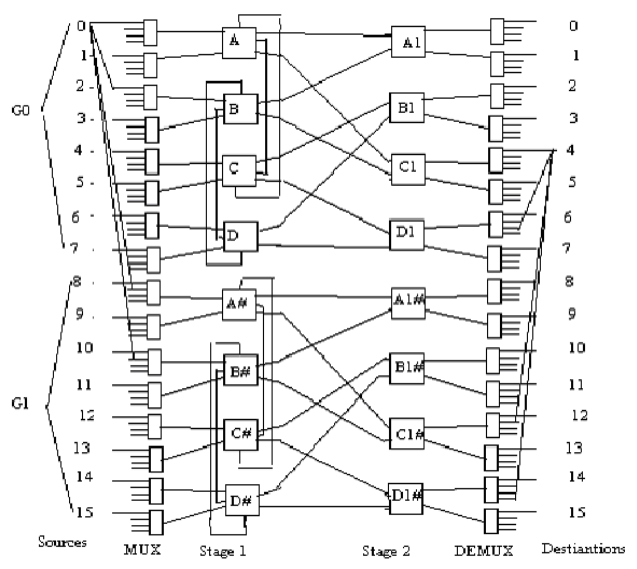

Figure 1: An MABN of size 16 X 16.

\subsection{Construction of IMABNs}

IMABN (Irregular modified baseline network) is a modified augmented baseline network with one additional stage,increase 
size of switch and more auxiliary links. To construct an IMABN of size $\mathrm{N}$ i.e. $\mathrm{N}$ sources and $\mathrm{N}$ destinations, two identical groups of N/2 sources and N/2 destinations need to be formed first. Each source is linked to both the groups via multiplexers. There is one $4 \times 1$ MUX for each input link of a switch in stage 1 and one $1 \mathrm{x}$ 4 DEMUX for each output link of a switch in stage n-1. IMABN consists of two identical sub-networks which are denoted by $\mathrm{G}^{\mathrm{i}}$ switches A, B, C, D belonging to stage 1 of a subnetwork $\left(\mathrm{G}^{\mathrm{i}}\right)$ form a conjugate subset, switches $\mathrm{A}$ and $\mathrm{B}$ form a conjugate pair, and switches $\mathrm{A}$ and $\mathrm{C}$ form a conjugate loop.Thus an IMABN of size $\mathrm{N}$ consists of $\mathrm{N}$ number of $4 \times 1$ MUXs, $N$ number of 1 x 4 DEMUXs, and $n-2$ stages of N/2 switches each (the last stage has $2 \times 2$ switches and the remaining stages have $3 \times 3$ switches) and one stage with $\mathrm{N} / 8$ switches. Observe that this construction procedure has two benefits. First, the network can tolerate the failure of any switch in the network. And,secondly it provides a topology which lends itself to on-line repair and maintainability, as a loop can be removed from any stage of the IMABN without disrupting the operation of the network. Since the sub-networks are identical, so the VLSI implementation of the network becomes simple. IMABN of size 16x16 is shown Fig 2.

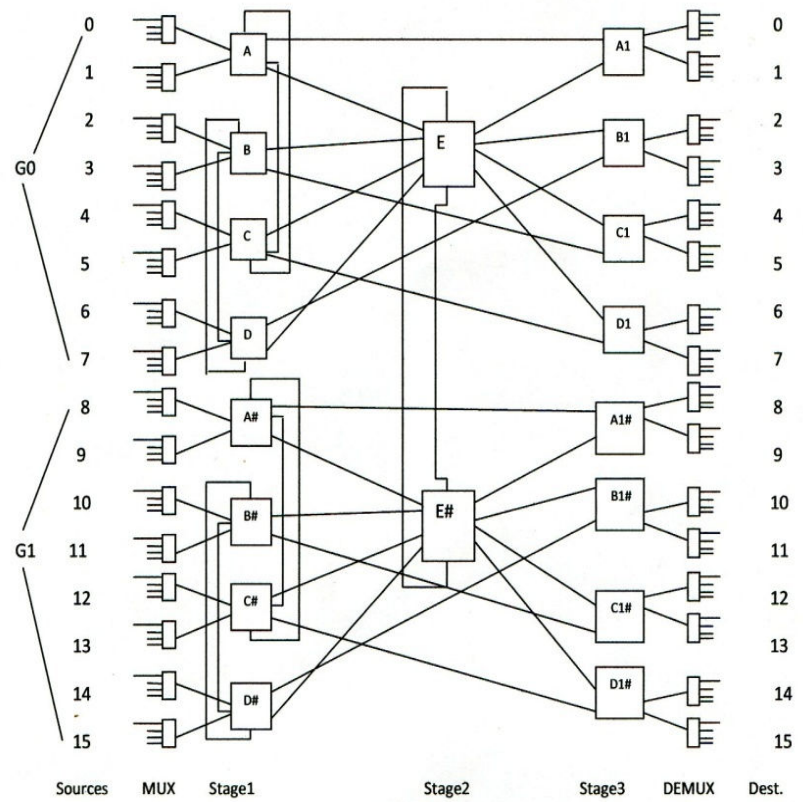

Figure 2: An IMABN of size 16x16

\section{RELIABILITY ANALYSIS}

Reliability is one of the most important parameter to measure the performance of MINs. It is calculated in terms of MTTF. MTTF is the expected time elapsed before some source is disconnected from some destination. To analyze the MTTF, the following assumptions will used:-

1) switch failures occur independently in a network with a failure rate of $\lambda$ (a reasonable estimate for $\lambda$ is about $10^{-6}$ per hour).
2) Failure of the multiplexers and demultiplexers also occur independently with failure rates of $\lambda_{m}$ and $\lambda_{d}$ receptively, which can be different from $\lambda$.

Reliability equations for proposed IMABN is derived for both lower bound and upper bound in terms of MTTF is given below:-

\subsection{Upper Bound(Optimistic):}

In IMABN each source is connected to two multiplexers in each sub-network, and each switch has a conjugate. So if we assume that the IMABN is operational as long as one of the two multiplexers attached to a source (in a particular sub-network) is operational and as long as a conjugate pair (loop or switch) is not faulty, then we will permit as many as one half of the components to fail and the IMABN may still be operational. This permits a simple reliability block diagram of the optimistic (upper) bound as shown in Figure 3.

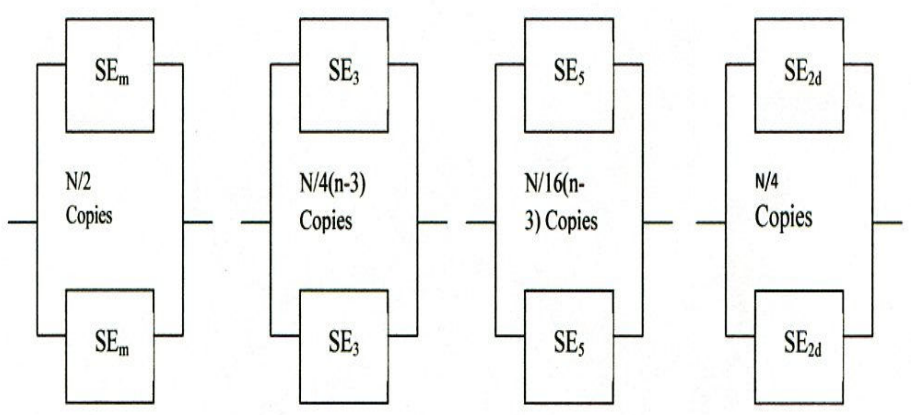

Figure 3: Reliability block diagram of IMABN for MTTF upper bound

$\mathrm{R}_{\text {IMABN-ub }}(\mathrm{t})=\mathrm{fl} * \mathrm{f} 2 * \mathrm{f} 3 * \mathrm{f} 4$

$$
\begin{aligned}
& f 1=\left[1-\left(1-e^{-\lambda_{m} t}\right)^{2}\right]^{(N / 2)} \\
& f 2=\left[1-\left(1-e^{-\lambda_{3} t}\right)^{2}\right]^{N / 4(n-3)}
\end{aligned}
$$$$
f 3=\left[1-\left(1-e^{-\lambda_{5} t}\right)^{2}\right]^{N / 16(n-3)}
$$$$
f 4=\left[1-\left(1-e^{-\lambda_{2 d} t}\right)^{2}\right]^{N / 4}
$$

Where $\lambda_{\mathrm{m}=} \lambda, \lambda_{3=} 2.25 \lambda, \lambda_{5}=6.25 \lambda, \lambda_{2 \mathrm{~d}=} 3 \lambda$

$\operatorname{MTTF}_{\text {IMABN-ub }}=\int_{0}^{\infty} \mathrm{R}_{\text {IMABN-ub }}(\mathrm{t}) \cdot \mathrm{dt}$ 


\subsection{Lower Bound (Pessimistic):}

At the input side of the IMABN, the routing scheme does not consider the multiplexers to be an integral part of a $3 \times 3$ switch. For example, as long as at least one of the two multiplexers attached to a particular switch is operational, the switch can still be used for routing. Hence, if we group two multiplexers with each switch in the input side and consider them a series system $\left(\mathrm{SE}_{3 \mathrm{~m}}\right)$, then we will have a conservative estimate of the reliability of these components. Their aggregate failure rate will be $\lambda_{3 \mathrm{~m}}=4.25 \lambda$. Finally these aggregated components and the switches in the intermediate stages can be arranged in pairs of conjugate loops. To obtain the pessimistic (lower) bound on the reliability of IMABN, we assume that the network is failed whenever more than one conjugate loop has a faulty element or more than one conjugate switch in the last stage fails. The reliability block diagram is shown in Figure 4.
Table 1: MTTF of MABN and IMABN for different network size

\begin{tabular}{|c|c|c|c|c|}
\hline LOGN & MABN_LB & MABN_UB & IMABN_LB & IMABN_UB \\
\hline 4 & 4.953677 & 5.184155 & 5.626158 & 5.784969 \\
\hline 5 & 4.733636 & 4.959739 & 5.368372 & 5.46313 \\
\hline 6 & 4.522473 & 4.74445 & 5.1316 & 5.190016 \\
\hline 7 & 4.317438 & 4.535628 & 4.908977 & 4.943551 \\
\hline 8 & 4.116806 & 4.331575 & 4.69608 & 4.713475 \\
\hline 9 & 3.919452 & 4.131159 & 4.49002 & 4.494246 \\
\hline 10 & 3.724616 & 3.933593 & 4.288884 & 4.282583 \\
\hline
\end{tabular}

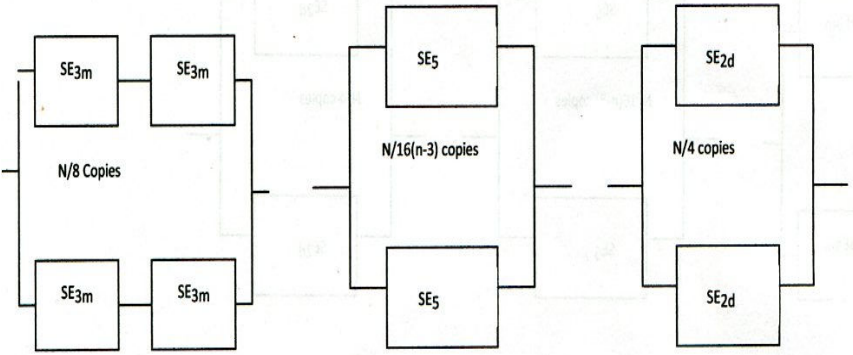

Figure 4: Reliability block diagram of IMABN for MTTF lower bound.

$\mathrm{R}_{\mathrm{IMABN}-\mathrm{lb}}(\mathrm{t})=\mathrm{f} 1 * \mathrm{f} 2 * \mathrm{f} 3$

$$
\begin{aligned}
& f 1=\left[1-\left(1-e^{-2 \lambda_{3 m} t}\right)^{2}\right]^{(N / 8)} \\
& f 2=\left[1-\left(1-e^{-\lambda_{5} t}\right)^{2}\right]^{N / 16(n-3)} \\
& f 3=\left[1-\left(1-e^{-\lambda_{2 d} t}\right)^{2}\right]^{(N / 4)}
\end{aligned}
$$

Where $\lambda_{3 \mathrm{~m}=} 4.25 \lambda, \lambda_{3}=2.25 \lambda, \lambda_{5}=6.25 \lambda, \lambda_{2 \mathrm{~d}=} 3 \lambda$

$\mathrm{MTTF}_{\text {IMABN-lb }}=\int_{0}^{\infty} \mathrm{R}_{\text {IMABN-lb}}(\mathrm{t}) \cdot \mathrm{dt}$

The results of MTTF reliability equations have been shown in Table1:-

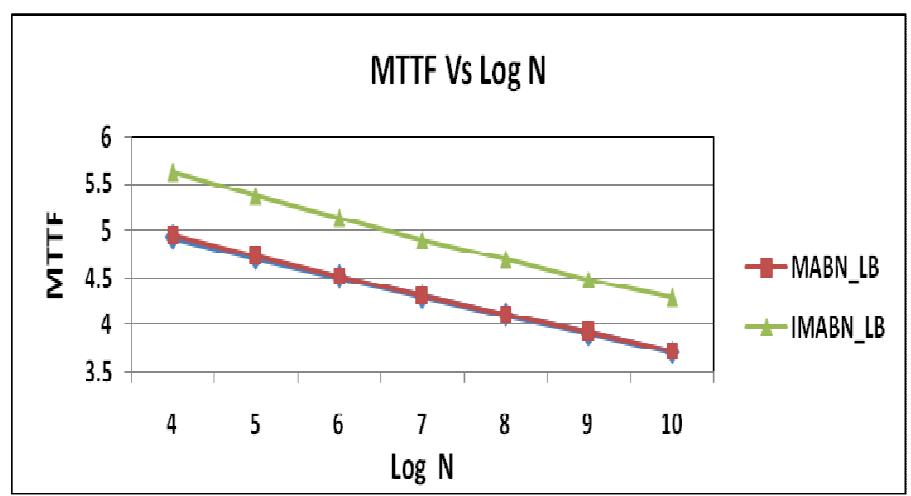

Figure 6: MTTF( Lower Bound) Comparison of MABN and IMABN.

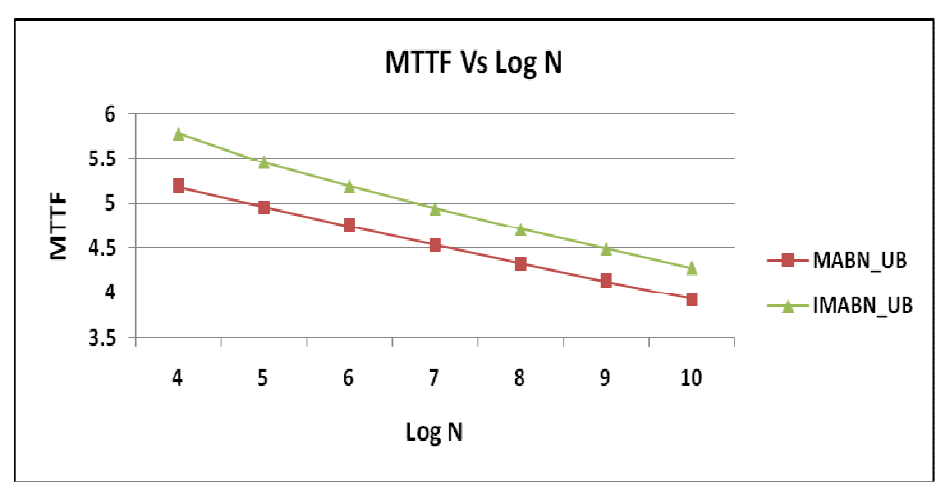

Figure 7: MTTF( Upper Bound) Comparison of MABN and IMABN. 


\section{COST-EFFECTIVENESS}

To estimate the cost of the network, one common method is to calculate the switch complexity with an assumption that the cost of a switch is proportional to the number of gates involved, which is roughly proportional to the number of cross-points within a switch .For example a $2 \times 2$ switch has four units of hardware cost, whereas a 3 × 3 switch has nine units. Cost of $\mathrm{MABN}$ and IMABN for different network size is shown in Table2.

Table 2: Cost of MABN and IMABN for different network size

\begin{tabular}{|c|c|c|}
\hline $\log \mathbf{N}$ & MABN & IMABN \\
\hline 4 & 2.365488 & 2.447158 \\
\hline 5 & 2.735599 & 2.781037 \\
\hline 6 & 3.096215 & 3.120574 \\
\hline 7 & 3.449633 & 3.462398 \\
\hline 8 & 3.797406 & 3.804003 \\
\hline 9 & 4.140634 & 4.144013 \\
\hline 10 & 4.480122 & 4.481844 \\
\hline
\end{tabular}

Now a simple measure of the cost-effectiveness for reliability can be given by comparing MTTF and the cost of the network. The cost-effectiveness of MABNs and IMABNs (both upper and lower bounds) are evaluated and compared, and the improvement in results is shown in a Table 3. From the results we can observe that IMABNs are more cost effective than most of the other fault-tolerant networks.

Table 3: MTTF per unit $\log$ Cost Vs $\log N$

\begin{tabular}{|c|c|c|c|c|}
\hline $\begin{array}{c}\text { Log } \\
\mathbf{N}\end{array}$ & MABN_LB & MABN_UB & IMABN_LB & IMABN_UB \\
\hline 4 & 2.0941459 & 2.1915795 & 2.29905793 & 2.36395402 \\
\hline 5 & 1.7303837 & 1.8130358 & 1.930349 & 1.9644219 \\
\hline 6 & 1.4606457 & 1.5323387 & 1.64444105 & 1.66316069 \\
\hline 7 & 1.2515644 & 1.3148146 & 1.41779686 & 1.42778242 \\
\hline 8 & 1.08411 & 1.1406668 & 1.23451007 & 1.23908288 \\
\hline 9 & 0.9465826 & 0.9977117 & 1.08349564 & 1.08451542 \\
\hline 10 & 0.8313649 & 0.8780102 & 0.95694629 & 0.9555404 \\
\hline
\end{tabular}

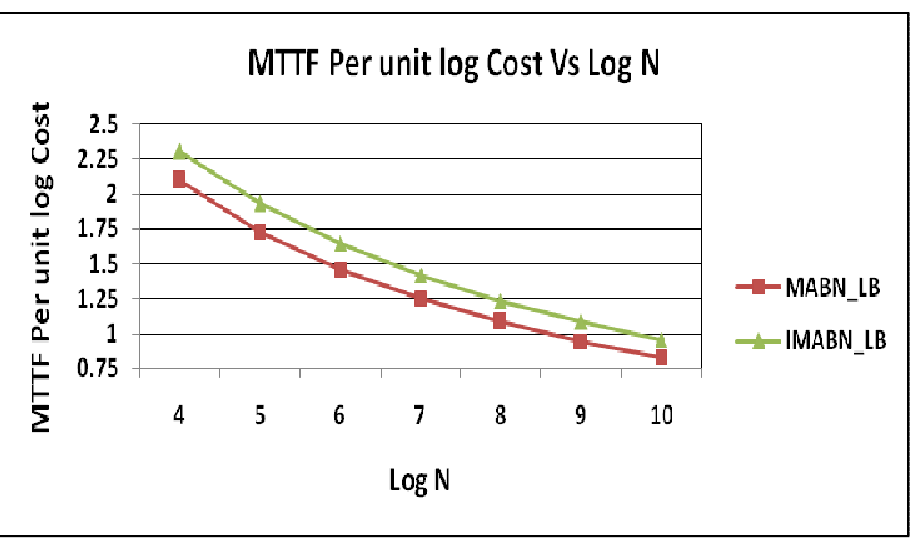

Figure 8: Comparison of MTTF(Lower Bound) Per unit log Cost Vs Log N

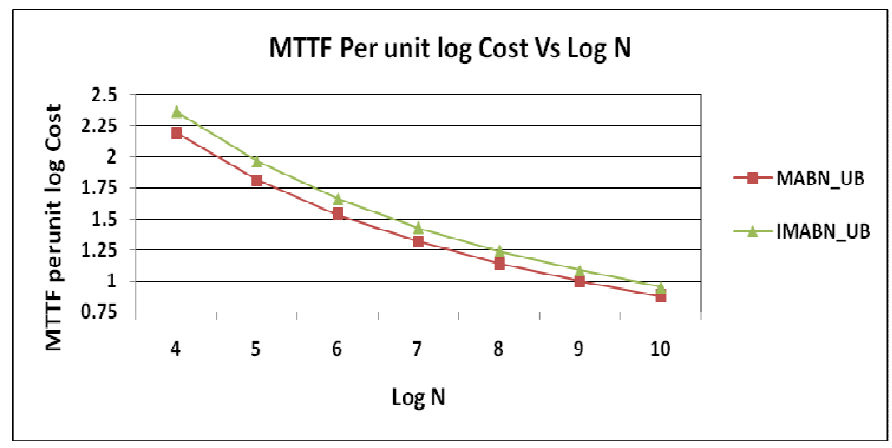

Figure 9: Comparison of MTTF(Upper Bound) Per unit log Cost Vs Log N

\section{CONCLUSION}

In this paper, we proposed and analyzed new irregular Fault-tolerant multistage interconnection network named as Irregular modified baseline network(IMABN). IMABN is dynamically re-routable and providing multiple paths of varying lengths between source and destination pairs. It has been found that IMABN has six possible paths whereas in MABN has only four. The analysis of the lower and upper bounds of MTTF showed that IMABN is having better reliability than other related fault-tolerant networks.However, if such reliability comes at the expense of high cost,it may have little value in practice.Our analysis on the cost of networks showed that IMABNs is generally,more cost-effective than MABN.Reliability analysis and cost study shows that IMABN has better performance than MABN.This paper also shows that variable reliability for the different paths chosen in case of irregular MINs and constant reliability in case or regular MINs.The number of switching elements encountered is varying depending on the path chosen which makes the average rate of failure of the network to be lesser for the irregular MINs as compared to that of regular 
MINs. A critical examination of this MINs corroborates that considering the delay and reliability of fault-tolerant MINs, the irregular networks are a better candidate to be used in parallel processing environments.

\section{REFERENCES}

[1] Bhuyan Laxmi N., Yang Qing and Aggarwal P. Dharma, "Performance of Multiprocessor Interconnection Networks", Proceeding of IEEE, February 1989, pp. 25-37.

[2] Bhogavilli Suresh K. and Abu-Amara Hosame, "Design and Analysis of High Performance Multistage Interconnection Networks", IEEE Transactions on Computers, vol. 46, no. 1, January 1997, pp. 110 -117.

[3] Bansal P.K, Singh Kuldeep and Joshi R.C, “ On Fault tolerant Multistage Interconnection Network", Conference on Computer Electrical Engineering, vol. 20, no.4, 1994, pp. 335-345.

[4] Blaket James T. and Trivedi Kishor S., "Reliabilities of Two Fault-Tolerant Interconnection Networks", Proceeding of IEEE, 1988, pp. 300-305.

[5] Charles Chenggong Charles and Bruck Jehoshua, "Tolerating Multiple Faults in Multistage Interconnection Networks with Minimal Extra Stages", IEEE Transactions on Computers, vol. 49, no. 9, September 2000, pp. 998-1004.
[6] Cheema Karamjit, Aggarwal Rinkle, "Design Scheme and Performance Evaluation of new Fault-Tolerant Multistage Interconnection Network", IJCSNS International Jounal of Computer Science and Network Security , VOL.9 NO.9,September 2009.

[7] Nitin, "On Analytic Bounds of Regular and Irregular Fault-tolerant Multi-stage Interconnection Networks", Proceedings of International Conference, 2006.

[8] Sadawarti Harsh and Bansal P.K., “ Fault Tolerant Irregular Augmented Shuffle Network", Proceeding of the 2007 WSEAS International Conference on Computer Engineering and Applications, Australia, January 17-19,2007. pp. 7-12.

[9] Sengupta J. and Bansal P.K, "Performance of Regular and Irregular Dynamic MINs", Proceeding of International Conference IEEE TENCON, 1999, pp. 427-430.

[10] Sharma Sandeep and Bansal P.K., "A New Fault Tolerant Multistage Interconnection Network", Proceeding of International Conference IEEE TENCON, 2002, pp. 347-350. 